\title{
ColabSaber: Um framework de suporte pedagógico na construção colaborativa de saberes
}

\author{
Waldir Siqueira Moura ${ }^{1}$, Juliana B. S. França ${ }^{1,2}$, Angélica F. S. Dias ${ }^{1,4}$, Jonice de \\ Oliveira Sampaio ${ }^{1}$, Marcos R. S. Borges ${ }^{1,3}$
}

${ }^{1}$ Programa de Pós-graduação em Informática (PPGI) - Universidade Federal do Rio de Janeiro

(UFRJ) - Rio de Janeiro - RJ - Brasil

${ }^{2}$ Departamento de Computação (DECOMP) - Universidade Federal Rural do Rio de Janeiro UFRRJ - Seropédica - RJ - Brasil

${ }^{3}$ TECNUN, University of Navarra, Donostia/San Sebastián, Spain.

${ }^{4}$ Núcleo de Computação Eletrônica (NCE) - Universidade Federal do Rio de Janeiro (UFRJ) Rio de Janeiro - RJ - Brasil

waldirsmeufrj.br, julibsf@gmail.com, angelica@nce.ufrj.br, joniceldcc.ufrj.br, mborgeseppgi.ufrj.br

\begin{abstract}
This paper proposes the ColabSaber Framework, which aims to change the traditional format of lecture-based classes with the use of the learning principles defended by Vygotsky, such as language, culture and social interaction. ColabSaber uses the fundamentals of Group Storytelling as educational support for elementary school students. This paper points out the importance of the collaborative construction of comic books as an intermediate support in the traditional teaching-learning process, also considering the technological advances and the ease of students in handling new technologies. As a result, the article concludes a significant improvement in the academic performance of the participating students and a motivational element of the academic engagement of the participating students.
\end{abstract}

Resumo. O presente artigo propõe o Framework ColabSaber que tem por objetivo associar ações de aulas expositivas com os princípios de aprendizagem defendidos por Vygotsky, sendo eles: linguagem, cultura e interação social na aprendizagem. O ColabSaber usa os fundamentos do Group Storytelling como apoio educacional para alunos do Ensino Fundamental. O artigo aponta a importância da construção colaborativa das histórias em quadrinhos como apoio intermediário no processo de ensino-aprendizagem tradicional, considerando também os avanços tecnológicos e a facilidade dos alunos no manuseio das novas tecnologias. Como resultado, o artigo apresenta melhor rendimento escolar dos alunos participantes e elemento motivacional do engajamento acadêmico dos alunos participantes.

\section{Introdução}

Nos últimos anos, as escolas têm enfrentado dificuldades na implementação de tecnologias como ferramentas de apoio e estímulo à aprendizagem tanto fora quanto dentro das salas de aula. Existem vários fatores que podem dificultar essa aprendizagem. Segundo Martin et al. (1996) essas dificuldades encontradas estariam relacionadas a dificuldade dos alunos de colocarem em prática as rotinas, planejamentos e controle de processos cognitivos envolvidos na realização de uma dada atividade.

O uso de histórias como ferramenta didática tem sido um meio de minimizar essas 
dificuldades [Gouvêa, et al., 2016]. Os alunos passam horas concentrados em cinema e mídias sociais, mas não conseguem focar sua atenção por quarenta minutos em sala de aula. Isso acontece porque as histórias, nos cinemas e nas mídias sociais, são contadas de formas mais interessantes. Por isso, a técnica Storytelling pode ser uma solução para essa aprendizagem em sala de aula.

Segundo Mcsill et al. (2013) o Storytelling é definido como o velho hábito de contar histórias. Desde nossos ancestrais, o ato de se reunir em volta de uma fogueira para, ao fim de cada dia, contar sobre o que foi vivenciado ao decorrer dele é a forma mais eficaz de perpetuar a cultura; as práticas e conhecimentos culturais tão importantes para a sobrevivência de grupos, ou seja, Storytelling é a arte de contar uma história por meio da palavra escrita, da música, da mímica, das imagens, do som ou dos meios digitais como afirma Borges (2011).

Esta pesquisa propõe o Framework de Suporte Pedagógico na Construção Colaborativa de Saberes (ColabSaber). Este método tem por objetivo unir ações de aprendizagem tradicional em conjunto com iniciativas que defendam os princípios de aprendizagem defendidos por Vygotsky - linguagem, cultura e interação social. A essência do ColabSaber está na consolidação e construção de novos saberes a partir de práticas colaborativas de construção e contação de Histórias em Quadrinhos (HQ) sobre o tema da disciplina escolar. O objetivo principal desta investigação é propor uma alternativa de apoio pedagógico, que colabore com o processo de aprendizagem do aluno através de estímulos, e melhora de seu rendimento acadêmico. Para esta investigação, duas turmas do ensino fundamental e particular brasileiro foram submetidas a um experimento com consentimento da escola e de seus pais. As histórias em quadrinhos, apresentadas e discutidas neste artigo, foram criadas por alunos participantes do experimento conduzido com o uso da ferramenta tecnológica Pixton ${ }^{1}$.

Este artigo é organizado considerando na seção 1 a introdução e motivação desta pesquisa, explorando os conceitos de Storytelling e Group StoryTelling (GST). Na seção 2 exploramos os fundamentos teóricos do trabalho colaborativo em sala de aula e os trabalhos relacionados com o tema; na seção 3 explicitamos a estrutura metodológica e o método adotado para esta pesquisa; na seção 4 apresentamos o framework desenvolvido como proposta ao trabalho colaborativo; na seção 5 discutimos os passos para o desenvolvimento do experimento; na seção 6 são apresentados os resultados obtidos e na seção 7 fazemos nossas considerações finais.

\section{Storytelling e Group Storytelling}

Storytelling é o ato de contar histórias. Silva et al. (2018) diz que a contação de histórias era inicialmente feita para transmitir e compartilhar conhecimentos entre os homens primitivos. No contexto escolar o storytelling tem sido aplicado para investigar métodos de ensino [Salim et al., 2016] e [Lage et al., 2017], e também, para estimular a criatividade e curiosidade do aluno nas aulas para que, deste modo, se tornem mais participativos e interessados [Robin, 2019]. Uma história pode ser contada por uma pessoa ou por um grupo (Group Storytelling), como expõe Borges (2011).

\footnotetext{
${ }^{1}$ https://www.pixton.com/br/ - Link acessado em 01/07/2020.
} 


\subsection{Trabalhos relacionados}

Histórias em quadrinho tem sido um forte recurso de suporte à educação. Esse ferramental tem sido aplicado na contação de histórias e, portanto, observa-se claramente uma referência direta com a teoria de Storytelling. A literatura destaca algumas pesquisas que defendem o uso de histórias em quadrinho para suporte pedagógico, como ferramentas de inclusão e facilitação da aprendizagem. Para Silvério (2006), as histórias em quadrinhos são entendidas como aspectos educadores e auxiliadores no trabalho com a leitura. Costa (2011) defende as HQs aplicadas em sala de aula. Segundo ele, esse recurso tem permitido aos docentes unir texto, gramática e literatura de forma agradável, fomentando o engajamento de alunos no aprendizado da língua portuguesa. Severo e Severo (2015) seguem a mesma linha de pesquisa de Costa (2011) discutindo o uso de HQs como recurso didático na melhoria do processo de ensino-aprendizagem. Para eles, as HQs além de estimular a troca de conhecimentos entre os alunos, ainda auxilia no hábito de leitura, desenvolvendo a criatividade, a autonomia e a criticidade dos alunos.

De acordo com [Kotujansky, 2009], as histórias em quadrinhos podem ser um recurso para contribuir com a formação inicial de professores que atuarão nos anos iniciais de ensino fundamental, no contexto da disciplina de Ciências por meio da produção de materiais educacionais digitais (MED) com apoio da ferramenta ToonDoo Maker. Piconi e Tanaka (2003) discutem o uso de HQs na educação como estratégia de inclusão de alunos. Esta pesquisa utiliza o software HagáQuê na tentativa de auxiliar o desenvolvimento de alunos autistas em seu processo de interação e comunicação com a sociedade. Em [Campos et al., 2004] é discutida a inclusão durante o processo de aprendizagem de surdos através do sistema SIGNHQ desenvolvidos para a criação e leitura de histórias em quadrinhos com suporte à língua de sinais.

Essas pesquisas apresentadas preveem a introdução de histórias em quadrinhos no processo educacional, seja na construção de recursos didáticos, seja no processo de aprendizagem do aluno. Algumas delas ainda discutem aspectos de inclusão para alunos. Neste artigo pretendemos discutir a aplicação de histórias em quadrinhos mediada por recursos tecnológicos com a finalidade de estimular o processo de aprendizagem do aluno e melhorar o rendimento em provas. Busca-se nesta pesquisa aplicar as HQs como reforço do conteúdo de uma disciplina escolar que tenha sido passado de forma expositiva. $\mathrm{Na}$ próxima seção será apresentada e discutida a estrutura metodológica deste artigo, o tipo de pesquisa aplicado e o Framework ColabSaber.

\section{Estrutura Metodológica e Método}

A presente pesquisa tem por essência características de pesquisa qualitativa e quantitativa. Ao retornar para o objetivo principal deste artigo, temos a Construção do Framework ColabSaber.

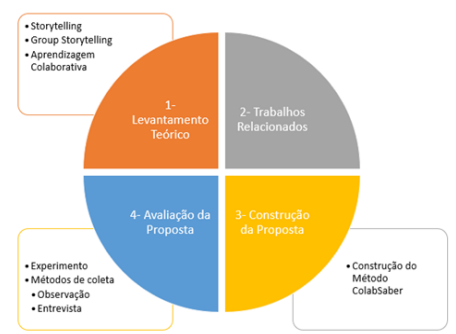

Figura 1: Estrutura metodológica. 
Quanto aos objetivos específicos, destacam-se o estímulo do processo de aprendizagem do aluno e a melhoria do rendimento em provas. Dessa maneira, o Framework ColabSaber foi construído (subseção a seguir); é um experimento planejado e executado com turmas do ensino básico de uma escola particular a fim de verificar se os objetivos da pesquisa foram alcançados.

Como método de coleta de dados foi aplicada a observação e entrevistas. Os instrumentos de coleta de dados foram: histórias produzidas pelas crianças participantes, anotações das observações e entrevistas, e notas em provas sobre o conteúdo ministrado. Detalhes sobre o experimento serão apresentados mais adiante neste artigo. A Figura 1 apresenta o desenho esquemático da metodologia científica aplicada no artigo.

\section{Framework de suporte pedagógico na construção colaborativa de saberes - ColabSaber}

O Framework ColabSaber (Figura 02) é resultado da fase 3 da estrutura metodológica da pesquisa (Figura 01). Este método tem por princípio consolidar estratégias de aprendizagem colaborativa com as aulas expositivas tradicionais na intenção de estimular o engajamento dos alunos em aula e melhorar o aprendizado de cada um deles.

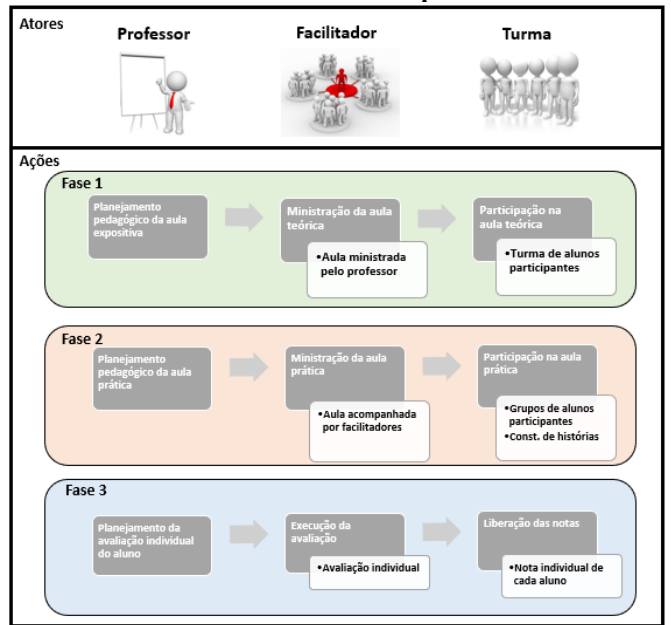

\section{Figura 2: Estrutura do Framework ColabSaber}

O ColabSaber é fundamentado nos princípios da aprendizagem colaborativa. Segundo Castro e Menezes (2011), os principais princípios para a estruturação da aprendizagem colaborativa são: os estudantes trabalham juntos buscando aprender e estes são responsáveis não apenas por sua própria aprendizagem, mas também pela as dos demais. Estes princípios implicam em metas coletivas e potencializam as possibilidades de aprendizagem dos alunos à medida que são atendidos. O ColabSaber tem por princípio estender o método tradicional de ensino com a proposta de integrar ações da aprendizagem construtivista nesta iniciativa. Para isso, aulas práticas com recursos tecnológicos e ações de construção de histórias em quadrinhos.

O ColabSaber é estruturado em três fases e suas ações são executadas pelo professor da disciplina, facilitador, e turmas de alunos. A primeira fase é composta por três atividades gerais. O Planejamento pedagógico da aula expositiva é realizado pelo professor da disciplina de acordo com a ementa e planejamento do curso. Além disso, este precisa estar orquestrado com a segunda fase do ColabSaber a fim de que a aula prática, através da construção colaborativa de histórias (com o uso da técnica Group 
Storytelling), possa ser realizado pelos alunos. A ministração da aula teórica é uma atividade realizada pelo mesmo professor que a planejou.

A fase 2 dedica esforços ao planejamento, execução e participação da aula prática. Neste planejamento espera-se que o professor junto ou não do facilitador prepare o ambiente, seja ele tecnológico ou não. A condução das aulas será acompanhada pelo facilitador que pode ou não ser o professor da disciplina. Nesta aula, espera-se que os alunos sejam capazes de construir e contar histórias cujo enredo seja a aula teórica que participaram anteriormente. Para a condução dessas aulas, a turma deverá ser organizada em grupos de trabalho. A formação dos grupos poderá seguir o melhor critério na visão do facilitador. Um exemplo de método para a formação de grupos de trabalho está em (Tardelli et al., 2019).

Nesta proposta, espera-se que a aula prática atue como um reforço ao conteúdo apresentado na aula expositiva. Além disso, espera-se que o grupo construa saberes em conjunto e que deficiências de alguns integrantes possam ser supridas pelos demais. $\mathrm{O}$ facilitador tem por papel orientar a construção das histórias em quadrinhos através de narrativas textuais e desenhos, sanar eventuais dúvidas, mas sem interferir ativamente no conteúdo construído pelos alunos. Com este princípio prático, apresentamos o ColabSaber pautado nos seguintes conceitos educacionais defendidos por Vygotsky 2016): Interação social da linguagem e da cultura na aprendizagem. Ou seja, a aprendizagem é um resultado adaptativo que tem natureza social, histórica e cultural.

O ColabSaber defende que a construção de saberes a partir de histórias em quadrinhos é uma forma do aluno internalizar novos conhecimentos a partir de referências culturais de seu grupo que foram compartilhadas na sessão da aula prática. Além disso, as HQs são estruturadas em uma linguagem própria para alunos do ensino básico. Por fim, a Fase 3 prevê a condução de avaliações a fim de investigar o rendimento individual dos alunos que participaram da instância do ColabSaber.

\section{Contextualização da investigação e preparação do experimento}

Ao acompanhar o rendimento de alunos do sétimo e oitavo ano (Ensino Fundamental II) de uma escola particular brasileira, situada em Lorena - SP, foi constatado que $50 \%$ dos alunos do sétimo ano e $65 \%$ dos alunos do oitavo ano apresentaram desempenho abaixo da média esperada na disciplina de Ciências. O uso de HQs para o Ensino Fundamental II é justificado pois os Parâmetros Curriculares Nacionais - PCNs - preveem sua utilização como recurso didático-pedagógico. Diante desta realidade, foi aplicado o ColabSaber com a participação de 38 alunos em um experimento.

O experimento foi estruturado considerando a participação de duas turmas. Cada uma participou em duas fases: aula expositiva sobre o conteúdo de Ciências "Desastre Ambiental" e aula de reforço. Metade de cada turma participou de um reforço tradicional e expositivo em sala de aula, enquanto a outra metade das turmas foi direcionada para o Laboratório de Informática a fim de trabalharem o conceitual da aula expositiva com a construção de histórias colaborativas sob supervisão do professor. Neste estudo foi aplicada a ferramenta Pixton de construção de HQ. Nela são disponibilizados cenários, personagens e tipos de quadros que podem ser alterados com alguns cliques do mouse.

\section{Planejamento}

Tendo como base o PCN, duas séries do ensino Fundamental II foram escolhidas para a aplicação do Group Storytelling apoiado na ferramenta de história em quadrinhos Pixton.

O primeiro contato com a direção do Colégio ocorreu em 2 de maio de 2019. Após a aprovação da diretora os pesquisadores, juntamente com a coordenação pedagógica e 
professores de Ciências e Informática, iniciaram a organização detalhada de como seria feita a aplicação e do número de aulas que precisariam ter disponíveis. A aula expositiva sobre "Desastre Ambiental" seguiu a programação normal de conteúdo previsto pela escola.

Ainda como atividade de preparação para o experimento, a aula expositiva foi preparada pelo professor de Ciências com antecedência e apresentada previamente para os pesquisadores. Com base na aula preparada os pesquisadores dividiram o conteúdo em cinco partes. Cada grupo de alunos seria responsável por relatar, através da técnica group storytelling representada em quadrinhos, um trecho da aula. Após a execução do experimento, alguns participantes foram entrevistados a fim de coletarmos suas impressões a respeito das práticas de reforço sobre o conteúdo "Desastre Ambiental". O experimento conduzido na presente pesquisa ocorreu nos dias 30/05/2019 e 31/05/2019 e foi organizado de acordo com as atividades apresentadas abaixo:

Atividades de preparação: Coletar as notas desses alunos na disciplina no trimestre anterior; organizar o experimento em conjunto com direção e professores de Ciências e Informática; dividir os alunos da $7^{\mathrm{a}}$ e $8^{\mathrm{a}}$ séries em duas turmas aleatoriamente.

Atividades de execução: dia 1 - Aula coletiva para as duas turmas com o professor de ciências; dia 1 - Aula introdutória ao Pixton no laboratório para T2; dia 2 - Aula de reforço em sala de aula para a T1; dia 2 - Aula de reforço no laboratório utilizando o Pixton com a T2; dia 2 - Prova sobre o conteúdo para as turmas T1 e T2; dia 2 -Correção das provas pelo professor de Ciências; dia 2 - Entrevista com alunos da T2 e professores sobre a experiência com Pixton.

\section{Execução da Investigação}

O primeiro dia de condução do experimento ocorreu das $7 \mathrm{~h} 40$ às $8 \mathrm{~h} 40$ com a aula coletiva no auditório do Colégio com a presença das duas séries. Nela o professor de Ciências apresentou o conteúdo programado previamente, com o auxílio do projetor, e tirou eventuais dúvidas a respeito do conteúdo. Após a explanação foi solicitado-lhe que fizesse a divisão dos grupos e encaminhasse alguns para a sala de Informática e direcionasse os outros para a aula de Artes.

A divisão dos grupos foi feita de forma aleatória, assim o sétimo ano foi dividido em dois grupos, sendo o primeiro com os respectivos números de chamada ímpares $(1,3$, $5,7,9,11,13,15,17)$ e o segundo de números de chamada pares $(2,4,6,8,10,12,14$, $16,18)$, assim obtivemos dois grupos iguais com nove alunos cada. A divisão dos grupos do oitavo ano seguiu o mesmo critério e gerou dois grupos, um dos alunos de números ímpares $(1,3,5,7,9,11,13,15,17,19)$ com 10 alunos e outro com os alunos de números pares $(2,4,6,8,10,12,14,16,18,20)$ com 10 alunos também. Assim, decidimos que os grupos formados por alunos de números ímpares fariam a aula tradicional enquanto os grupos de alunos de números pares participariam do experimento com o Pixton no laboratório. Após a separação dos grupos, os alunos de números pares seguiram para o Laboratório de Informática onde foram subdivididos nos respectivos grupos: alunos do $7^{\circ}$ ano $\{2,4,6\}$ grupo $1 ;\{8,10,12\}$ grupo 2 e $\{14,16$ e 18$\}$ grupo 3 . Alunos do $8^{\circ}$ ano $\{2,4,6,8,10\}$ grupo 4 e $\{12,14,16,18,20\}$ grupo 5 .

Os recortes da aula para a construção colaborativa de histórias em quadrinhos foram organizados pelo professor da disciplina. No entanto, o professor não soube quais seriam os grupos que fariam cada recorte. A organização dos grupos é apresentada a seguir: Grupo 1 - Equilíbrio e desequilíbrio ecológico; Grupo 2 - Barragem de Mariana; 
Grupo 3 - Poluição; Grupo 4 - Recursos renováveis e não renováveis e o Grupo 5 Sustentabilidade.

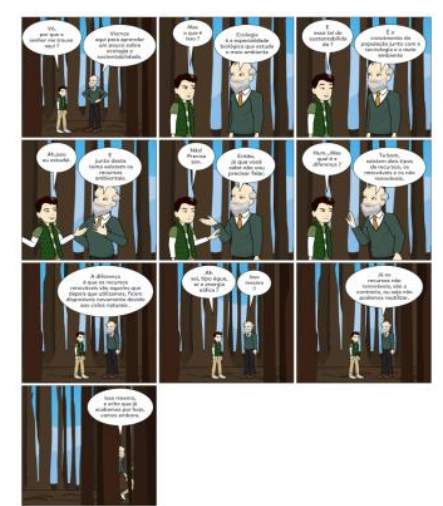

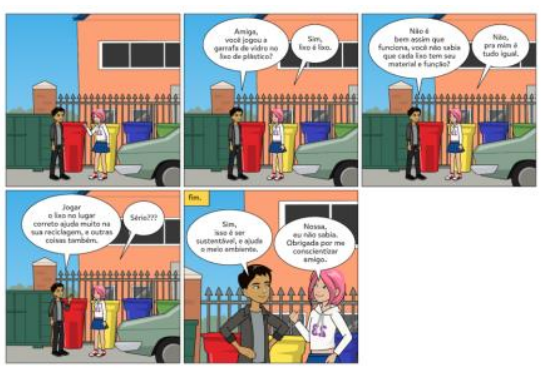

Figura 4: HQ - grupo 5.

Figura 3: $\mathrm{HQ}$ - grupo 4.

Ainda no primeiro dia, sem que os alunos soubessem seus grupos e seus fragmentos, o professor de Informática começou às $9 \mathrm{~h}$ uma aula para apresentar o Pixton; ele fez uma explanação utilizando o projetor para demonstrar como fazer a criação dos quadrinhos para todos os grupos de números pares e depois permitiu que todos usassem o software e tirassem suas dúvidas. A aula de apresentação do software aconteceu sem nenhum contratempo, e todos conseguiram utilizá-lo com tranquilidade.

No segundo dia o experimento seguiu sua aplicação a partir das 7 h50 quando teve início a aula de reforço no auditório para os alunos dos grupos ímpares e a aula de reforço com o software no Laboratório de Informática para os alunos dos grupos de números pares. A aula de reforço tradicional transcorreu tranquilamente até às $8 \mathrm{~h} 50$ quando terminou e iniciou-se a avaliação. Já na aula no laboratório ocorreu um contratempo com a rede de internet, mas logo foi corrigido para a continuidade do experimento. Às $10 \mathrm{~h} 40$ foi iniciada a avaliação deste grupo. A avaliação que todos os alunos foram submetidos foi idêntica e elaborada pelo mesmo professor da disciplina, ela continha dez questões dissertativas, valendo um ponto cada.

\section{Resultados Obtidos}

Para avaliação dos resultados, foram realizadas análises quantitativa e qualitativa, considerando todas as informações coletadas na pesquisa prévia e a opinião dos alunos, professores e responsáveis pelo colégio.

As análises quantitativas foram conduzidas com os grupos que geraram as Figuras 3 e 4, em especial com a comparação das notas de cada aluno (reforço tradicional e reforço através das narrativas em quadrinhos). A preparação do experimento teve início com a coleta do histórico de notas dos alunos que participaram e com isso foi elaborado uma tabela para as duas salas para que, após a aplicação da avaliação tradicional e do Storytelling, pudéssemos fazer a comparação dos dados. Após essa coleta os alunos foram selecionados e distribuídos em dois grupos - como explicado anteriormente. Após a preparação, as instruções e a aula tradicional de Ciências, os grupos foram divididos e, enquanto um fez a aula de reforço tradicional, o outro fez a aula de reforço com o auxílio do Storytelling. 


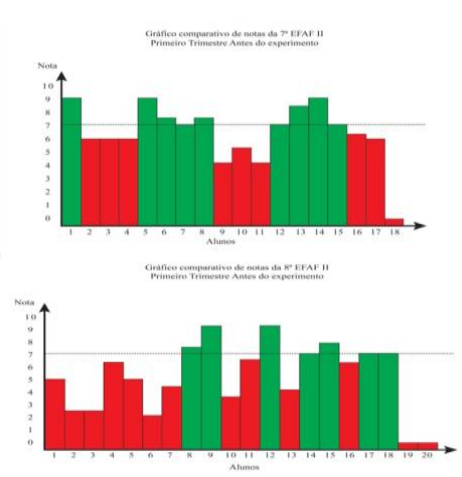

Figura 5: Notas em
ciências antes do
experimento.

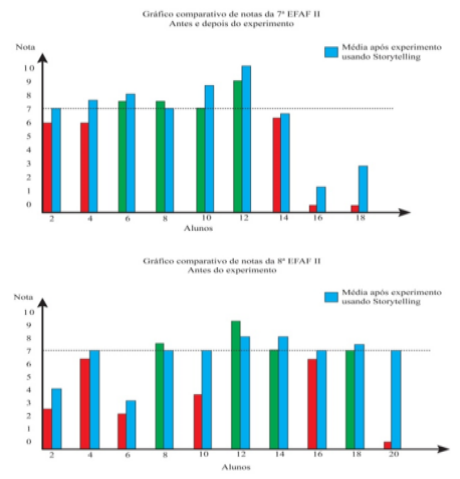

Figura 6: Comparativo de notas.

A Figura 5 apresenta os resultados obtidos pelas turmas de Ciências em provas antes da execução do experimento. Nelas é possível observar menos de 50\% de aproveitamento dos alunos. As barras apresentadas em vermelho revelam alunos que não atingiram a nota média na disciplina. Já os gráficos de Figura 6 apresentam os resultados das mesmas turmas de $7^{\circ}$ e $8^{\circ}$ anos após a participação no experimento proposto por esta pesquisa. Os gráficos da Figura 6 apresentam uma melhora significativa dos alunos participantes do experimento em laboratório (uso do Pixton e aplicação do Group Storytelling). No $8^{\circ}$ ano, apenas dois alunos não atingiram a média, enquanto no $7^{\circ}$ ano apenas três alunos não atingiram a média sete. A análise qualitativa teve por objetivo confirmar os resultados alcançados na análise quantitativa. Como conclusão obtida pela percepção dos alunos que foram submetidos à aula de reforço com as narrativas, é possível afirmar que todos eles manifestaram satisfação ao trabalhar com a ferramenta e gostaram do método de aula utilizado. Os trechos a seguir confirmam essa conclusão: "Foi legal, foi bem interessante, mostra os ensinamentos. Ajudou bastante a mexer com o computador e memorizar o conteúdo, essas coisas. E ajuda a gente a se esforçar mais.".

Os professores envolvidos com o experimento, assim como os responsáveis pela administração do Colégio se mostraram bastante satisfeitos com o desempenho da atividade. Segundo eles: "Foi uma experiência gratificante, esse tipo de atividade é sempre algo enriquecedor para ambos os lados, mas especialmente, para os estudantes..."; "Eu achei que foi muito especial. Essas atividades causam um movimento bom no Colégio, eles ganham e nós também..."

\section{Considerações finais e trabalhos futuros}

Neste artigo foi proposto o Framework ColabSaber que une ações de aulas expositivas tradicionais com os princípios da aprendizagem colaborativa pautada nos três pilares da aprendizagem de Vygotsky: linguagem, cultura e interação social na aprendizagem. $O$ ColabSaber foi avaliado através de um experimento a fim de observar se sua estrutura, uma vez implementada em sala de aula, é capaz de estimular os alunos academicamente e melhorar o rendimento escolar dos alunos participantes. Para isso, o experimento considerou duas turmas onde uma delas teve apenas aulas expositivas e a segunda participou também de uma aula prática de construção colaborativa de histórias em quadrinhos.

Para análise desta investigação, foi realizada uma análise comparativa de dados relacionando as notas e médias dos alunos em relação ao desempenho na avaliação da 
disciplina após o experimento. Nesta análise as evidências apresentadas nas notas demonstram que a construção colaborativa de histórias em quadrinhos sobre o tema da aula colabora com o processo de construção do conhecimento, isto porque ambas as turmas, tradicional e de aplicação do ColabSaber, tiveram uma melhora no desempenho do experimento. A média das avaliações dos alunos que participaram do experimento executando o método ficou em 8.8 enquanto a média das avaliações dos alunos que participaram das aulas tradicionais ficou em 5.7. Desta forma, esse estudo apresenta indícios sobre a melhoria no aprendizado fomentada pela aplicação da técnica de construção de narrativas em sala de aula. Foi observado nesta pesquisa que a construção de histórias em quadrinhos pelos alunos favorece a absorção do conhecimento para avaliações acadêmicas tradicionais.

$\mathrm{Na}$ análise qualitativa neste estudo, foi observado que os alunos se sentiram satisfeitos com a atividade de construção de quadrinhos, onde as narrativas que compõem o Group Storytelling foram produzidas. Eles se sentiram motivados em trabalhar no laboratório de Informática criando suas histórias baseadas no conteúdo programático de Ciências apresentado em sala de aula. Foi observado também que a atividade de construção colaborativa de histórias usando o Pixton apoiou os alunos na consolidação dos conceitos da aula anterior.

Como trabalho futuro, faremos o mesmo experimento com outras turmas a fim de confirmar os resultados alcançados, e também novos experimentos com o objetivo de investigar a aplicação de técnicas de enturmações focando no conhecimento coletivo.

\section{References}

Borges, Marcos. (2011). Conhecimento coletivo. In Sistemas Colaborativos. Pimentel, Mariano; Fuks, Hugo. (Orgs). Rio de Janeiro: Elsevier. P. 198.

Castro, Alberto; Menezes, Crediné. (2011). Aprendizagem colaborativa com suporte computacional. In Sistemas Colaborativos. Pimentel, Mariano; Fuks, Hugo. (Orgs). Rio de Janeiro: Elsevier. P. 135.

Colaço, V. De F. R. 2004. Processos interacionais e a construção de conhecimento e subjetividade de crianças. In Psicologia: Reflexão e Crítica, Porto Alegre, V. 17, N. 3, P. 333340.

Damiani, Magda Floriana. (2008). Entendendo o trabalho colaborativo em educação e revelando seus beneficios. In Educar, Curitiba, N. 31, P. 213-230, Editora UFPR.

de Borba Campos, M., Maiocchi, D., \& Bortolin, M. (2004, November). SIGNHQ: sistema de autoria para criação e leitura de Histórias em Quadrinhos com suporte à Língua de Sinais. In Brazilian Symposium on Computers in Education (Simpósio Brasileiro de Informática na Educação-SBIE) (Vol. 1, No. 1, pp. 44-46).

Gouvêa, Maria Teresa; Pimentel, Mariano; Santoro, Flavia Maria; Cappelli, Claudia. 2016. Externalização do conhecimento através de group storytelling: um estudo de caso em tutorial online. In XII Brazilian Symposium on Information Systems, Florianópolis, Sc, May 17-20.

Kotujansky, Silvio. (2009). Um modelo para a elaboração colaborativa de conteúdos didáticos digitais que utilizem a metáfora de histórias em quadrinhos e recursos hipermídia. Dissertação defendida na Universidade Federal de Santa Catarina como requisito para obtenção do grau de Mestre.

Lage, B. B., França, J. B. S., Dias, A. F. S. Vivacqua, A. S., Borges, M. R. S. (2017). Aplicando o group storytelling no compartilhamento de experiências e na avaliação em sala de aula. In VI Congresso Brasileiro de Informática na Educação (CBIE 2017). Recife, Porto Alegre. Anais do XXIII Workshop de Informática na Escola (WIE 2017), 2017. 
IX Congresso Brasileiro de Informática na Educação (CBIE 2020)

Anais do XXVI Workshop de Informática na Escola (WIE 2020)

Martin, E; Marchesi, A. (1996). Desenvolvimento metacognitivo e problemas de aprendizagem. Porto Alegre: Artes Médicas. P. 41.

Mcsill, James. (2013). 5 Lições de storytelling: fatos, ficção e fantasia. 1. Ed. São Paulo: Dvs Editora. In Santos, Leonardo Schwertner Dos. Storytelling: O Poder da Narrativa Estratégica Dentro do Branding e Marketing. P. 5.

Neves, Sílvia Da Conceição. (2012). A História em quadrinhos como recurso didático em sala de aula. Trabalho de Conclusão de Curso de Artes Visuais, Habilitação em Licenciatura, do Departamento de Artes Visuais da Universidade de Brasília. Palmas.

Piconi, A. C., \& Tanaka, E. H. (2003, November). A construção de histórias em quadrinhos eletrônicas por alunos autistas. In Brazilian Symposium on Computers in Education (Simpósio Brasileiro de Informática na Educação-SBIE) (Vol. 1, No. 1, pp. 385-394).

Robin, B. (2016). Digital storytelling: A powerful technology tool for the 21 st century classroom. Disponível Em: Http://Digitalstorytellingclass.Pbworks.Com/F/Digital+Storytelling+A+Powerful.Pdf . Acesso em: 29 jun. 2019. Torres, Niquelle Leite; Soares, Tathiana Santos; Conceição, Fábio Henrique Gonçalves. Dificuldade de Aprendizagem: Além do Muro Escolar In: II Encontro Científico Multidisciplinar da Faculdade Amadeus.

Salim, R., Dias, A. F. S., França, J. B. S., Lage, B. B., Borges, M. R. S. (2016). O uso de storytelling para apoiar o compartilhamento de conhecimento. In $13^{\circ}$ Simpósio Brasileiro de Sistemas Colaborativos (SBSC). Porto Alegre. v. 1.

Salvador, Coll. Palacios, J.; Marchesi, A. (Org.). (1996). Desenvolvimento psicológico e educação. Porto Alegre: Artes Médicas. V. 2.

Silva, Alexsandra Monteiro Da; Correa, Ana Claudine Siqueira; Filho, José Mário Ribeiro De Souza; Farias, Gleybson Dos Santos; Souza, Ricardo André Cavalcante De. (2018). Gestão do conhecimento como ferramenta para melhoria do trabalho coletivo e colaborativo. In Ijkem, Int. J. Knowl. Eng. Manage. V.7, N.17 • Florianópolis, Sc • Mar. 2018/Jun. 2018. (Ufsc) Patricia De Sá Freire E João Artur De Souza (Ed.), P. 65-83.

Silva, Deivid Eive Dos Santos; Corrêa, Marialina Sobrinho; Lima, Celson Pantoja. (2018). Criação de storytelling digital aplicado a disciplina redação com os alunos das $3^{a}$ séries do Ensino Médio. In Revista Tecnologias na Educação - Ano 10 - Número/Vol.25 - julho.

Tancicleide Gomes; Fernanda Castro, Patricia Tedesco. (2017). Desenvolvendo o pensamento computacional na educação infantil: Um toolkit educacional sobre conceitos de programação baseado em storytelling transmedia. In Nuevas Ideas en Informática Educativa TISE. Volumen 13, p. 31 - 40. Santiago de Chile.

Tardelli, André; França, Juliana; Dias, Angélica; Vivacqua, Adriana; Borges, Marcos. (2019). A influência da personalidade do aluno na Construção de Grupos de Trabalho em Sala de Aula. In Anais do Simpósio Brasileiro de Informática na Educação - SBIE.

Torres, Patrícia Lupion; Irala, Ersom Adriano F. (2014). Aprendizagem colaborativa: teoria e prática. In Coleção Agrinho, N. 3, P. 61-93, Editora Agrinho.

Silvério, Luciana Begatini Ramos. (2006) O valor pedagógico das histórias em quadrinhos no percurso do docente de língua portuguesa. I Jornada de didática: o ensino como foco. CEMAD - Paraná.

Costa, Marsoniel Felipe da. (2011). Os quadrinhos em sala de aula. Artigo científico apresentado à Coordenação do Curso de Letras da Universidade Estadual da Paraíba - UEPB - Campus III como requisito para obtenção do grau de licenciado em Letras, habilitação I.

Severo, Marta F. da Silva; Severo, David Ferreira. (2015). As HQs como ferramenta pedagógica em sala de aula. Artigo publicado na revista CESMAC edição digital de 2015. https://cesmac.edu.br/revistas-digitais

Vigotski, L. S. (1996). Pensamento e linguagem. Martins Fontes: São Paulo. 